\title{
Molecular biomarkers in current management of metastatic colorectal cancer
}

\section{Suebpong Tanasanvimon}

Division of Medical Oncology, Department of Internal Medicine, Faculty of Medicine, Chulalongkorn University, King Chulalongkorn Memorial Hospital, Thai Red Cross Society, Bangkok 10330, Thailand.

Correspondence to: Dr. Suebpong Tanasanvimon, Division of Medical Oncology, Department of Internal Medicine, Faculty of Medicine, Chulalongkorn University, King Chulalongkorn Memorial Hospital, Thai Red Cross Society, 1873 Rama IV rd. Pathumwan, Bangkok, Bangkok 10330, Thailand. E-mail: surbpong@yahoo.com

How to cite this article: Tanasanvimon S. Molecular biomarkers in current management of metastatic colorectal cancer. $J$ Cancer Metastasis Treat 2018;4:57. http://dx.doi.org/10.20517/2394-4722.2018.38

Received: 15 Jun 2018 First Decision: 9 Jul 2018 Revised: 21 Aug 2018 Accepted: 24 Oct 2018 Published: 21 Nov 2018

Science Editor: Guo-Feng Xie Copy Editor: Cai-Hong Wang Production Editor: Zhong-Yu Guo

\begin{abstract}
Over the past two decades, the treatment outcomes in metastatic colorectal cancer (mCRC) have been remarkably improved, largely from the evolution of systemic therapy. Also, the molecular biomarkers have played a major role in this improvement by their predictive value in current treatment paradigm in mCRC. Currently, extended RAS mutation analysis is required for consideration of anti-epidermal growth factor receptor therapy in patients with mCRC. Several uncommon gene alterations have emerged as the potential targets for their matched molecular targeted therapy. Although, most patients with $\mathrm{mCRC}$ do not derive benefit from immunotherapy. By using microsatellite instability or mismatch repair test, we are now able to identify a small subgroup of patients with mCRC who have a very good response to immune checkpoint inhibitors. With the increasing number of required biomarkers in $\mathrm{mCRC}$ management, multiplex gene panel testing is now replacing single gene testing strategy. In patients accessible to matched molecular targeted therapy, especially for clinical trials, the comprehensive genomic profiling might be the preferred testing method. Although, it is potentially benefit in mCRC treatment, the liquid biopsy is not yet clinically applicable. The optimal utilization of molecular biomarker testing is required for best treatment outcomes in individual patients.
\end{abstract}

Keywords: Molecular biomarkers, metastatic colorectal cancer, treatment

\section{INTRODUCTION}

Over the past two decades, the treatment outcomes in metastatic colorectal cancer (mCRC) have been significantly improved, largely because of the evolution of systemic therapy. Although chemotherapy is

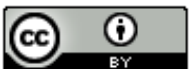

(C) The Author(s) 2018. Open Access This article is licensed under a Creative Commons Attribution 4.0 International License (https://creativecommons.org/licenses/by/4.0/), which permits unrestricted use, sharing, adaptation, distribution and reproduction in any medium or format, for any purpose, even commercially, as long as you give appropriate credit to the original author(s) and the source, provide a link to the Creative Commons license, and indicate if changes were made. 
still the mainstay treatment in mCRC, its efficacy could be significantly enhanced by the biologic therapies including anti-angiogenesis and anti-epidermal growth factor receptor (anti-EGFR) agents. In a selected subgroup, the median overall survival is now up to 40 months with current treatment paradigm ${ }^{[1]}$. Recently, the molecular targeted therapy and immunotherapy have been demonstrated as the emerging effective therapeutic options for some patients with mCRC. The molecular biomarker plays a critical role as a tool for personalized therapy in current and upcoming treatment paradigm in patients with mCRC. The optimal utilization of molecular biomarker testing is required for best treatment outcomes in individual patients. This article reviews clinical application and limitation of current and emerging biomarkers in management of mCRC.

\section{BIOMARKERS FOR ANTI- EGFR THERAPY}

EGFR is a transmembrane receptor tyrosine kinase playing a major role in carcinogenesis of several cancers including CRC. Although the EGFR expression was required for patients to be eligible in the initial anti-EGFR trials in $\mathrm{mCRC}^{[2,3]}$. The later reports demonstrated poor correlation between EGFR expression and treatment response ${ }^{[4,5]}$. Instead, KRAS mutation is a robust negative predictor for benefit of anti-EGFR in patients with mCRC. However, not all patients with wild-type KRAS mCRC will have benefit from firstline chemotherapy and anti-EGFR combination therapy, patient selection for anti-EGFR therapy has been evolved through biomarker analysis in subsequent clinical trials.

\section{RAS}

RAS protein is a critical regulator of growth factor-induced cell proliferation and survival in both cancer and normal cells. There are three RAS family genes including KRAS, NRAS and HRAS. KRAS mutation is found in $30 \%-40 \%$ of CRC. NRAS mutation has been demonstrated in up to $3 \%$ of CRC while HRAS mutation was very rare in $\mathrm{CRC}^{[6]}$. In mCRC, KRAS exon 2 (codon 12 and 13) is the most frequent location for RAS mutation, with prevalence of $40 \%$. Other RAS mutations were found at KRAS exon 3 and 4 , and NRAS exon 2, 3 and 4, with prevalence of $15 \%-20 \%$. Totally, the prevalence of all RAS mutations was around 55\%-60\% in patients with $\mathrm{mCRC}^{[7,8]}$. The mutations promote constitutive activation of GTP-bound RAS, resulting in activation of downstream signaling pathways especially the RAF/MEK/ERK pathway and PI3K pathway.

As a key downstream regulator of EGFR pathway, the activated mutation of KRAS might be able to abrogate the anti-EGFR treatment effects. In 2008, a retrospective analysis of KRAS exon2 mutation of a phase III trial, CO.17, demonstrated that cetuximab improved overall survival (OS) and progression free survival (PFS) only in patients with wild-type KRAS tumors, not in patients with mutant KRAS tumors ${ }^{[\rho]}$. This finding was subsequently confirmed in several cohorts of phase II and III trials of both available anti-EGFR agents including cetuximab and panitumumab ${ }^{[3,10,11]}$. In PRIME study, a prospective analysis of KRAS exon 2 mutation revealed a detrimental effect of additional panitumumab to chemotherapy for untreated patients with mutant KRAS mCRC ${ }^{[12]}$. In this cohort, a subsequent report demonstrated the extended analysis of RAS mutation, including KRAS and NRAS exon 2, 3 and 4, as the better predictive factor for panitumumab in patients with $\mathrm{mCRC}^{[13]}$. There was a detrimental effect of panitumumab in patients with wild-type KRAS exon2 with mutant other RAS mCRC. Similarly, this predictive effect of extended RAS mutation was subsequently confirmed in several phase II/III cetuximab and panitumumab trials. Therefore, the extended analysis of RAS mutation is required in selection of patients with mCRC for anti-EGFR therapy.

In contrast, KRAS mutation did not predict benefit of bevacizumab in patients with mCRC. In the analysis of phase III trial cohorts, additional bevacizumab to chemotherapy provides clinical benefit in both patients with wild-type and mutant KRAS mCRC ${ }^{[1,15]}$. Although patients with mutatnt KRAS mCRC seemed to live shorter than patients with wild-type KRAS mCRC in several anti-EGFR trials, prognostic 
value of KRAS mutation was confounded by the effectiveness of anti-EGFR therapy in patients with wildtype $K R A S^{[3,9-12]}$. There were conflicting results among the analysis in other RCT cohorts regarding the prognostic value of RAS mutation in $\mathrm{mCRC}^{[14,16]}$.

\section{BRAF}

As $B R A F$ is a key regulator in MAPK pathway, anti-EGFR therapy might not be effective in tumors with activated BRAF mutation. However, given the small number of patients with mutant BRAF tumors, the analysis of individual anti-EGFR randomized controlled trials did not consistently showed predictive effect of BRAF mutation for anti-EGFR therapy in patients with $\mathrm{mCRC}^{[13,17-20]}$. Recently, there were two metanalyses regarding predictive effect of $B R A F$ mutation for anti-EGFR treatment in patients with mCRC, although they showed no significant benefit of anti-EGFR therapy in patients with mutant BRAF mCRC. Pietrantonio et al. ${ }^{[21]}$ suggested BRAF mutation as a negative predictive biomarker for anti-EGFR therapy, while Rowland et al. ${ }^{[22]}$ concluded that there was insufficient evidence to exclude the benefit of anti-EGFR therapy in patients with mutant BRAF mCRC ${ }^{[21,22]}$. Although, there has been no definitive conclusion, the patients with mutant BRAF tumor are unlikely to derive treatment benefit from anti-EGFR therapy.

\section{Other biomarkers}

As one third of patients with wild-type RAS mCRC will not have objective response to first-line chemotherapy and anti-EGFR combined therapy. The additional potential biomarkers would help optimizing anti-EGFR therapy in mCRC.

PI3K-AKT-mTOR pathway is another key downstream signaling pathway of EGFR. Alterations of PIK3CA and PTEN were explored for its predictive value for anti-EGFR therapy in mCRC. In contrast to other RAS and BRAF, PIK3CA mutation and PTEN alterations are not mutually exclusive with KRAS exon 2 mutation. The prevalence of PIK3CA mutation was $4 \%-11 \%$ in patients with wild-type KRAS mCRC ${ }^{[19,23-27]}$. The prevalence of PTEN loss and mutation was 19\%-58\% and 7\%-9\%, respectively, in patients with wildtype KRAS mCRC ${ }^{[1,24-27]}$. For those patients with wild-type KRAS exon 2 mCRC, PIK3CA mutation and PTEN alterations were associated with poorer objective response rate and OS for anti-EGFR therapy in two metanalyses ${ }^{[28,29]}$. However, there might be different predictive effects between different PIK3CA mutations and different techniques detecting PTEN alterations.

EGFR ligands, epiregulin (EREG) and amphiregulin (AREG) are overexpressed in CRC ${ }^{[30,31]}$. EREG and AREG expressions in mRNA, tumor protein and plasma protein levels are associated with poor prognosis in $\mathrm{CRC}^{[31-33]}$. In vitro studies demonstrated their autocrine activation and reduction of cetuximab effect in AREG and EREG gene silencing CRC cells ${ }^{[34,35]}$. These preclinical studies led to several retrospective analyses demonstrating the correlation of EREG and AREG with anti-EGFR benefit in $\mathrm{mCRC}^{[25,36,37]}$. Most studies demonstrated association between high AREG and EREG mRNA expression and better survival in patients with CRC receiving anti-EGFR. In a metanalysis, these associations were still statistically significant only in patients with wild-type KRAS $\mathrm{mCRC}^{[38]}$. In a tumor analysis of CO.17 trial, the benefit of cetuximab was found only in high expression but not low expression of EREG mRNA in patients with wild-type KRAS mCRC ${ }^{[39]}$. This predictive effect was not shown in patients with mutant KRAS mCRC. Recently, a retrospective analysis of PICCOLO trial demonstrated similar predictive effect of AREG/ EREG mRNA expression for benefits of the additional panitumumab to irinotecan in patients with wildtype KRAS mCRC ${ }^{[40]}$. However, there are limitations for utility of AREG/EREG expression as a predictive biomarker for anti-EGFR including no standard cut off for these continuous variables and modest concordance between their expression in primary and metastatic sites. Although, the plasma levels of AREG and EREG might overcome these limitations, there have been limited data of their predictive value in patients with mCRC. 


\section{BIOMARKERS FOR IMMUNOTHERAPY}

Recently, immune checkpoint inhibitors including anti-programmed cell death-1 (PD-1)/PD-ligand 1 (PD-L1) and anti-cytotoxic T lymphocyte associate protein-4 (anti-CTLA-4), have emerged as a new treatment paradigm in several cancers especially non-small cell lung cancer and melanoma. Given the low tumor mutational burden, colorectal cancer was considered as a "cold" tumor for immune response. Correspondingly, the early anti-PD-1 trial revealed almost no response in mCRC. However, subsequent studies demonstrated high response rate in patients with mCRC with high microsatellite instability (MSI-H).

\section{MSI}

MSI-H or DNA mismatch repair (MMR) deficiency is found in $12 \%-15 \%$ of CRC. It is a hallmark phenotype of Lynch syndrome caused by germline mutation of MMR genes including $M L H_{1}, M S H_{2}$, MSH6 and PMS2. However, $80 \%$ of MSI-H/ deficient MMR (dMMR) CRC are sporadic and caused by epigenetic inactivation of MLH1. The prevalences of MSI-H/MMR deficiency are 20\%, 12\% and 5\% in CRC stage II, III and IV, respectively ${ }^{[1,42]}$. MSI-H/dMMR CRCs have distinct clinicopathologic features including rightsided location, poor differentiation, mucinous type and lymphocyte infiltration. To detect dMMR tumor, there are two diagnostic methods including MSI test and MMR protein immunohistochemical staining. MSI-H/dMMR is a good prognostic factor in early stage CRC, but patients with MSI-H/dMMR mCRC had poorer prognosis than patients with microsatellite stable (MSS) or proficient MMR (pMMR) mCRC ${ }^{[1,43,44]}$. Although MSI-H/dMMR may negatively predict the benefit of adjuvant fluouracil in stage II CRC, the metanalysis showed no significant difference in chemotherapy response rates between MSI-H/dMMR and MSS/pMMR mCRC ${ }^{[45]}$.

Currently, MSI-H/dMMR is the only predictive biomarker for immunotherapy in patients with mCRC. In early reports of anti-PD1 in human tumors, it seemed to be inactive in mCRC. However, 1 out of 33 patients with mCRC obtained complete response ${ }^{[46,47]}$. Given the hypermutated state and lymphocytic infiltration features of MSI-H/dMMR tumors, this particular subgroup has been explored for anti-PD-1 in mCRC. In a phase II trial, the response rates were $40 \%$ and $0 \%$ in patients with dMMR and pMMR mCRC, respectively ${ }^{[48]}$. Recently, the combination of anti-PD-1 and anti-CTLA-4, nivolumab and ipilimumab, had shown more robust treatment outcomes including response rate of $55 \%$ and 1 -yr PFS of $71 \%$ in previously treated patients with $\mathrm{dMMR} \mathrm{mCRC}^{[49]}$. Anti-PD-1 is now a standard treatment option in patients with MSI-H/dMMR mCRC.

\section{PD-L1}

PD-1 is expressed in activated mature T cells, while PD-L1 is constitutively expressed in tissue including tumor cells. Ligation of PD-1 and PD-L1 results in co-inhibitory signal repressing the T cell response. PD-L1 expression is currently the predictive and companion biomarker for anti-PD1 especially pembrolizumab in several cancers. In CRC, PD-L1 expression rate varied among several reports ${ }^{[50-52]}$. Although some reports showed higher PD-L1 expression in MSI-H CRC than MSS CRC ${ }^{[50-52]}$. This correlation was not consistent as reported by Droeser and colleagues in the largest study with 1,491 tumor samples ${ }^{[53]}$. These inconsistent findings in CRC might be largely caused by the variation of PD-L1 expression assessment including staining techniques, antibodies and scoring systems. Also there are some evidenced of temporal and spatial heterogeneity of PD-L1 expression in $\mathrm{mCRC}^{[54,55]}$. With these limitations and no evidence of its predictive effect for anti-PD1 therapy, there is still no clinical application of PD-L1 expression in patient with mCRC.

\section{Tumor mutational burden}

Recently, tumor mutational burden has become the potential predictive factor for anti-PD1 therapy in several cancers. Generally, CRC is considered low tumor mutational burden (TMB) cancer, but MSI-H/ dMMR CRC is constitutively high TMB tumor. As mentioned, MSI is very robust in predictive effect for anti-PD1 in mCRC. Moreover, MSI and MMR test is simple and less expensive than TMB assessment. 
Therefore, the clinical application of TMB is quite limited in patients with mCRC. Although, early report of TMB assessment by comprehensive genomic profiling (CGP) demonstrated $20 \%$ high TMB in MSS CRC, there was only $1 \%$ high TMB in MSS CRC in the subsequent study ${ }^{[56,57]}$. However, there were different cut off levels for high TMB among these studies, clinical validation of these cut points in association with benefit of anti-PD1 needed to be defined.

\section{BIOMARKERS FOR EMERGING TARGETED THERAPY}

Over the past decade, there have been several emerging molecular targeted therapies playing key role in cancer personalized therapy. In CRC, the most common genomic alterations including APC, RAS and TP53 are still undruggable. However, the current genomic profiling landscape leads to the discovery of uncommon targetable genomic alterations such as BRAF mutation, human epidermal growth factor receptor 2 (HER2) amplification and receptor tyrosine kinase (RTK) rearrangements in CRC.

\section{BRAF}

RAF protein is a key protein kinase transducing signal from RAS to MEK in MAPK pathway. BRAF mutation was found in $10 \%$ of $\mathrm{mCRC}^{[16,58,59]}$. BRAF $\mathrm{V} 600 \mathrm{E}$ is the most common mutation resulting in an increased activity of $B R A F^{[60]}$. In patients with mutant $B R A F$ CRC, there are distinct clinicopathological features including proximal tumor location, T4 tumor, poor differentiation and older age ${ }^{[61]}$. However, recently, there was a report of patients with mutant BRAF 594 or 596 with different features including rectal location, non-mucinous and no peritoneal metastasis. BRAF mutation is mutually exclusive with KRAS mutation but associated with $\mathrm{MSI}^{[59]}$.

$B R A F$ mutation is a poor prognostic factor. The analysis of phase III first-line chemotherapy studies in patients with mCRC demonstrated significantly shorter PFS and OS in patients with mutant BRAF tumors compared to wild-type BRAF tumors ${ }^{[16,41]}$. However, this prognostic effect was demonstrated only in patients with proficient MMR tumors ${ }^{[41]}$.

In contrast to melanoma, $B R A F$ targeted therapy did not have meaningful activity in patients with mutant $B R A F \mathrm{mCRC}{ }^{[62-65]}$. The preclinical study showed feedback activation of EGFR as a resistance mechanism to BRAF inhibitor in mutant BRAF mCRC ${ }^{[66]}$. Recently, a phase II trial showed significant improvement of PFS from 2.0 to 4.4 months and a response rate from $4 \%$ to $16 \%$ by additional vemurafenib to cetuximab and irinotecan in patients with mutant $B R A F \mathrm{~V} 600 \mathrm{mCRC}^{[6]]}$.The addition of MEK inhibitor or PI3K inhibitor to the dual therapy seemed to show better response rates and PFS ${ }^{[68,69]}$. Therefore, BRAF mutation is now an emerging target for combined BRAF inhibitor therapy in patients with mCRC.

\section{HER2}

HER2 is an EGFR family member activating MAPK and PI3K pathways. HER2 amplification/ overexpression is a prognostic and predictive marker for breast and gastric cancers. In CRC, it accounts for $2 \%-3 \%$ of $\mathrm{mCRC}$, but up to $5 \%$ in wild-type KRAS tumors ${ }^{[70]}$. It is very rare in patients with mutant $R A S / B R A F$ $\mathrm{mCRC}^{[70]}$. HER2 amplification/overexpression could be conventionally detected by in situ hybridization or immunohistochemical staining in tumor samples. The HER2 testing recommendation has been a consensus in breast and gastric cancers, but not in CRC. In a matched sample analysis, Lee and colleagues showed high discordance in positive results of FISH test between primary and metastatic sites ${ }^{[7]}$. There was also the possibility of changes in HER2 status after anti-EGFR therapy in patients with mCRC as shown in an analysis of plasma samples ${ }^{[72]}$. Moreover, there has been no consensus in diagnostic criteria for HER2 overexpression in CRC. The more stringent criteria including an intensely positive $>50 \%$ of cancer cells required for positivity by immunohistochemical staining was validated in HERACLES study, an anti-HER2 targeted study ${ }^{[73]}$. In this study, there was 30\% response rate to lapatinib and trastuzumab in patients with HER2 overexpressed mCRC. Another trial evaluating efficacy of combination of pertuzumab and trastu- 
zumab demonstrated $37.5 \%$ response rate in patients with HER2 overexpressed mCRC ${ }^{[7]}$. Though, HER2 is currently a predictive marker for the emerging anti-HER2 therapy in patients with mCRC. The optimal HER2 testing still needs to be defined in patients with mCRC.

\section{RTK rearrangement}

RTK rearrangements play a critical role in carcinogenesis of several cancers. These uncommon alterations are the emerging targets for novel effective therapies as demonstrated in ALK positive non-small lung cancer. Based on a few reports, RTK rearrangements are rare with prevalence of $0.2 \%-2.4 \%$ in CRC. Pietrantonio et al. ${ }^{[75]}$ had reported the clinicopathological analysis of 27 patients with ALK, ROS1 and NTRK gene rearrangement mCRC. As compared with 319 patients with no rearrangements, ALK, ROS1 and NTRK gene rearrangements were significantly more frequent in elderly patients with right sided, MSI-H and RAS/ $R A F$ wild type tumor. The study also demonstrated significantly shorter survival and poorer response to anti-EGFR in these patients with ALK, ROS1 and NTRK gene rearrangement ${ }^{[75]}$. By detection of these alterations, the patients could have benefit from the corresponding targeted therapy such as entrectinib in patients with CAD-ALK gene and LMNA-ETRK1 rearrangement ${ }^{[76-78]}$. However, given its rarity, the optimal diagnostic approach for these subgroups should be defined.

\section{CLINICAL SAMPLE FOR BIOMARKER ANALYSIS}

With the advancement of genomic analysis techniques, tumor genomic profiling is currently feasible in plasma samples. Although, tumor sample is still the gold standard for tumor genomic profiling, plasma sample or "liquid biopsy" addresses some limitations of tumor biomarkers.

\section{Tumor biomarkers}

Genomic profiling on tumor sample is the mainstay strategy for biomarker analysis in mCRC. However, there might be various available tumor sample sites, including primary tumor and metastatic sites. Primary tumor sample is more likely available in most patients with mCRC. The high concordance rates of genomic profiling of 90\%-100\% especially for RAS and BRAF mutations between primary and metastatic CRC samples were demonstrated in many studies ${ }^{[79-81]}$. Though, these high concordance rates have not been shown for uncommon genomic alteration, either primary or metastatic tumor was acceptable for genomic profiling in mCRC. For MSI/MMR, Haraldsdottir and colleagues showed perfect concordance of MMR status between primary tumor and metastasis, but a couple of reports showed up to $20 \%$ discordance rates ${ }^{[82-84]}$. Although, the spatial heterogeneity seems to be small in MCRC, the temporal heterogeneity, especially after treatment is potentially an issue for management in $\mathrm{mCRC}^{[85]}$. Therefore, the appropriate tumor samples for biomarker testing should be defined for the emerging genetic alterations and MSI/MMR tests, in order to maximize the benefit of molecular targeted agents and immune checkpoint inhibitors in mCRC.

\section{Liquid biopsy}

Not only a non-invasive and reproducible technique, but also a "liquid biopsy" would be able to overcome the limitation of tumor analysis including spatial and temporal heterogeneity. Currently, it is based on detection of circulating tumor DNA (ctDNA) by advanced technologies such as BEAMing method, droplet digital PCR or next generation sequencing (NGS). Several studies confirmed high concordance rate, 90\%$100 \%$, in $B R A F$ and KRAS mutations between tumor and liquid biopsy ${ }^{[86,87]}$. Two prospective studies demonstrated that early reduction in ctDNA during chemotherapy treatment could predict good responder in patients with $\mathrm{mCRC}^{[88,89]}$. Also, the emergence of KRAS mutation could be detected before radiographic disease progression during anti-EGFR therapy in patients with wild-type $K R A S \mathrm{mCRC}^{[85,90]}$. So, the liquid biopsy for disease monitoring during anti-EGFR therapy is potentially useful for clinical management of mCRC. However, the comprehensive gene analysis of ctDNA in mCRC is still not ready for clinical application, given the rarity of other than RAS targetable gene mutation and test sensitivity in mCRC. 
Table 1. Current application of biomarker in metastatic colorectal cancer

\begin{tabular}{|c|c|c|c|c|c|c|}
\hline Biomarker & Frequency & Clinical features & Predictive value & Current status & $\begin{array}{l}\text { Site of tumor } \\
\text { sample }\end{array}$ & $\begin{array}{l}\text { Detection } \\
\text { method` }\end{array}$ \\
\hline$R A S$ mutation & $55 \%-60 \%$ & None & $\begin{array}{l}\text { Resistance to anti- } \\
\text { EGFR therapy }\end{array}$ & $\begin{array}{l}\text { Standard } \\
\text { biomarker }\end{array}$ & $\begin{array}{l}\text { Primary tumor or } \\
\text { metastasis }\end{array}$ & $\begin{array}{l}\text { Single gene assay } \\
\text { Multiplex gene } \\
\text { panel assay } \\
\text { CGP }\end{array}$ \\
\hline$B R A F$ mutation & $10 \%$ & $\begin{array}{l}\text { Right-sided location, poorly } \\
\text { differention, elderly, } \\
\text { Wild-type RAS, MSI-H }\end{array}$ & $\begin{array}{l}\text { Resistance to anti- } \\
\text { EGFR therapy } \\
\text { Benefit of combination } \\
\text { BRAF inhibitors }\end{array}$ & $\begin{array}{l}\text { Standard } \\
\text { biomarker }\end{array}$ & $\begin{array}{l}\text { Primary tumor or } \\
\text { metastasis }\end{array}$ & $\begin{array}{l}\text { Single gene assay } \\
\text { Multiplex gene } \\
\text { panel assay } \\
\text { CGP }\end{array}$ \\
\hline MSI/MMR & $5 \%$ & $\begin{array}{l}\text { Right-sided location, } \\
\text { poor differentiation, } \\
\text { mucinous type, lymphocyte } \\
\text { infiltration }\end{array}$ & $\begin{array}{l}\text { Benefit of immune } \\
\text { checkpoint inhibitors }\end{array}$ & $\begin{array}{l}\text { Standard } \\
\text { biomarker }\end{array}$ & $\begin{array}{l}\text { No } \\
\text { recommendation }\end{array}$ & $\begin{array}{l}\text { MSI test } \\
\text { IHC } \\
\text { CGP }\end{array}$ \\
\hline \multicolumn{7}{|l|}{$\begin{array}{l}\text { Other rare genetic } \\
\text { alterations }\end{array}$} \\
\hline HER2 amplification & $2 \%-3 \%$ & None & $\begin{array}{l}\text { Benefit of anti-HER2 } \\
\text { therapy }\end{array}$ & Optional & $\begin{array}{l}\text { No } \\
\text { recommendation }\end{array}$ & $\begin{array}{l}\text { IHC } \\
\text { FISH } \\
\text { CGP }\end{array}$ \\
\hline RTK rearrangement & $0.2 \%-2.4 \%$ & $\begin{array}{l}\text { Right-sided location, } \\
\text { elderly, } \\
\text { MSI-H, wild-type RAS/RAF }\end{array}$ & $\begin{array}{l}\text { Benefit of RTK } \\
\text { inhibitors }\end{array}$ & Optional & No data & $\begin{array}{l}\text { FISH } \\
\text { CGP }\end{array}$ \\
\hline
\end{tabular}

${ }^{\star}$ Bold typing(s) is/are the preferred method(s). IHC: immuhistochemical staining; MSI: microsatellite instability; MSI-H: MSI-high; MMR: mismatch repair; EGFR: epidermal growth factor receptor; CGP: comprehensive genomic profiling; FISH: fluorescent in situ hybridization; HER2: human epidermal growth factor receptor 2; RTK: receptor tyrosine kinase

\section{MOLECULAR SUBTYPES OF COLORECTAL CANCER}

The genomic profiling has been widely performed in several types of cancers including CRC. In 2015, the CRC Subtyping Consortium analyzed and coalesced six independent classification systems into four consensus molecular subtypes, CMS 1-4, based on 3,962 patient samples ${ }^{[91]}$. However, less than $10 \%$ of these samples were mCRC, resulting in limitation of its clinical application in mCRC. Although, patients with CMS 4 had the worst overall survival and relapse free survival, patients with CMS1 had worst survival after relapse, corresponding to the findings of BRAF mutation and MSI-H as the poor prognostic factors in mCRC. In contrast to CMS1, patients with CMS2 had better survival after relapse than other subtypes, reflecting the good prognosis of wild-type RAS/BRAF mCRC. As current strategy in governance of systemic therapy is largely dependent on driver gene alterations, the molecular subtype is still not yet applicable in management of patients with mCRC.

\section{COMPREHENSIVE GENOMIC PROFILING}

With the advancement of molecular techniques such as NGS, the CGP is now available for clinical utility in management of patients with advanced cancer. Tumor CGP can provide insight into clinical relevant genetic alterations (CRGAs) guiding matched treatment selection for an individual patient. As described earlier, the current CRGAs in mCRC are RAS mutation, BRAF mutation and MSI-H, accounting for 75\% of all mCRC. All these alterations might be already known in the majority of patients with mCRC by sequential testing. Currently, CGP is used for detecting other rare CRGAs such as HER2 amplification, RTK rearrangement or other potential targets, and TMB assessment. However, this advantage of CGP is quite limited due to the rarity of these CRGAs and uncertain benefit of those matched therapeutic agents.

\section{CLINICAL APPLICATIONS OF BIOMARKERS IN MCRC}

As described above, most molecular biomarkers are currently used for treatment selection. For untreated patients with mCRC, RAS and BRAF mutations are required as the initial test for consideration of antiEGFR therapy. Although immune checkpoint inhibitors are not currently first-line therapy, MSI/MMR should also be included in those initial tests for a comprehensive treatment plan for each particular patient. Extended RAS mutation analysis including KRAS exon 2, 3, 4 and NRAS exon 2, 3, 4 is the standard test 
for evaluation $R A S$ status. In addition to anti-EGFR therapy consideration and prognostification, BRAF mutation is now the target for combination BRAF inhibitors. Other biomarkers such as HER2, RTK rearrangement or rare potential emerging targets were considered as beyond standard biomarkers. With the increasing number of required biomarkers in mCRC management, multiplex gene panel testing is now replacing single gene testing strategy. Of those patients accessible to matched molecular targeted therapy, especially for clinical trials, CGP might be the preferred testing method. Liquid biopsy is not yet clinical applicable in $\mathrm{mCRC}$, but there is potential benefit of the detection of drug resistance and dynamic change of biomarker status. The current application of biomarkers in mCRC was summarized in Table 1.

\section{DECLARATIONS}

\section{Authors' contributions}

Responsible for the paper, concept, design, literature search, and manuscript preparation, manuscript editing, manuscript revision: Tanasanvimon $\mathrm{S}$

\section{Availability of data and materials}

Not applicable.

\section{Financial support and sponsorship}

None.

\section{Conflicts of interest}

The author declared that there are no conflicts of interest.

\section{Ethical approval and consent to participate}

Not applicable.

\section{Consent for publication}

Not applicable.

\section{Copyright}

(c) The Author(s) 2018.

\section{REFERENCES}

1. Venook AP, Ou FS, Lenz HJ, Kabbarah O, Qu X, et al. Primary $\left(1^{\circ}\right)$ tumor location as an independent prognostic marker from molecular features for overall survival (OS) in patients (pts) with metastatic colorectal cancer (mCRC): analysis of CALGB/SWOG 80405 (Alliance). J Clin Oncol 2017;35:abstr3503.

2. Jonker DJ, O'Callaghan CJ, Karapetis CS, Zalcberg JR, Tu D, et al. Cetuximab for the treatment of colorectal cancer. N Engl J Med 2007;357:2040-8.

3. Van Cutsem E, Kohne CH, Hitre E, Zaluski J, Chang Chien CR, et al. Cetuximab and chemotherapy as initial treatment for metastatic colorectal cancer. N Engl J Med 2009;360:1408-17.

4. Chung KY, Shia J, Kemeny NE, Shah M, Schwartz GK, et al. Cetuximab shows activity in colorectal cancer patients with tumors that do not express the epidermal growth factor receptor by immunohistochemistry. J Clin Oncol 2005;23:1803-10.

5. Lenz HJ, Van Cutsem E, Khambata-Ford S, Mayer RJ, Gold P, et al. Multicenter phase II and translational study of cetuximab in metastatic colorectal carcinoma refractory to irinotecan, oxaliplatin, and fluoropyrimidines. J Clin Oncol 2006;24:4914-21.

6. Prior IA, Lewis PD, Mattos C. A comprehensive survey of Ras mutations in cancer. Cancer Res 2012;72:2457-67.

7. Ciardiello FL, Lenz HL, Kohne CH, Heinemann V, Tejpar S, et al. Treatment outcome according to tumor RAS mutation status in CRYSTAL study patients with metastatic colorectal cancer (mCRC) randomized to FOLFIRI with/without cetuximab. J Clin Oncol 2014;32:abstr3506.

8. Bokemeyer C, Kohne CH, Ciardiello F, Lenz HJ, Heinemann V, et al. FOLFOX4 plus cetuximab treatment and RAS mutations in colorectal cancer. Eur J Cancer 2015;51:1243-52.

9. Karapetis CS, Khambata-Ford S, Jonker DJ, O’Callaghan CJ, Tu D, et al. K-ras mutations and benefit from cetuximab in advanced colorectal cancer. N Engl J Med 2008;359:1757-65. 
10. Amado RG, Wolf M, Peeters M, Van Cutsem E, Siena S, et al. Wild-type KRAS is required for panitumumab efficacy in patients with metastatic colorectal cancer. J Clin Oncol 2008;26:1626-34.

11. Bokemeyer C, Bondarenko I, Makhson A, Hartmann JT, Aparicio J, et al. Fluorouracil, leucovorin, and oxaliplatin with and without cetuximab in the first-line treatment of metastatic colorectal cancer. J Clin Oncol 2009;27:663-71.

12. Douillard JY, Siena S, Cassidy J, Tabernero J, Burkes R, et al. Randomized, phase III trial of panitumumab with infusional fluorouracil, leucovorin, and oxaliplatin (FOLFOX4) versus FOLFOX4 alone as first-line treatment in patients with previously untreated metastatic colorectal cancer: the PRIME study. J Clin Oncol 2010;28:4697-705.

13. Douillard JY, Oliner KS, Siena S, Tabernero J, Burkes R, et al. Panitumumab-FOLFOX4 treatment and RAS mutations in colorectal cancer. N Engl J Med 2013;369:1023-34.

14. Hurwitz HI, Yi J, Ince W, Novotny WF, Rosen O. The clinical benefit of bevacizumab in metastatic colorectal cancer is independent of K-ras mutation status: analysis of a phase III study of bevacizumab with chemotherapy in previously untreated metastatic colorectal cancer. Oncologist 2009; 14:22-8.

15. Price TJ, Hardingham JE, Lee CK, Weickhardt A, Townsend AR, et al. Impact of KRAS and BRAF gene mutation status on outcomes from the phase III AGITG MAX trial of capecitabine alone or in combination with bevacizumab and mitomycin in advanced colorectal cancer. J Clin Oncol 2011;29:2675-82

16. Richman SD, Seymour MT, Chambers P, Elliott F, Daly CL, et al. KRAS and BRAF mutations in advanced colorectal cancer are associated with poor prognosis but do not preclude benefit from oxaliplatin or irinotecan: results from the MRC FOCUS trial. J Clin Oncol 2009;27:5931-7.

17. Karapetis CS, Jonker D, Daneshmand M, Hanson JE, O'Callaghan CJ, et al. PIK3CA, BRAF, and PTEN status and benefit from cetuximab in the treatment of advanced colorectal cancer--results from NCIC CTG/AGITG CO.17. Clin Cancer Res 2014;20:744-53.

18. Bokemeyer C, Van Cutsem E, Rougier P, Ciardiello F, Heeger S, et al. Addition of cetuximab to chemotherapy as first-line treatment for KRAS wild-type metastatic colorectal cancer: pooled analysis of the CRYSTAL and OPUS randomised clinical trials. Eur J Cancer 2012;48:1466-75.

19. Peeters M, Oliner KS, Parker A, Siena S, Van Cutsem E, et al. Massively parallel tumor multigene sequencing to evaluate response to panitumumab in a randomized phase III study of metastatic colorectal cancer. Clin Cancer Res 2013;19:1902-12.

20. Seymour MT, Brown SR, Middleton G, Maughan T, Richman S, et al. Panitumumab and irinotecan versus irinotecan alone for patients with KRAS wild-type, fluorouracil-resistant advanced colorectal cancer (PICCOLO): a prospectively stratified randomised trial. Lancet Oncol 2013;14:749-59.

21. Pietrantonio F, Petrelli F, Coinu A, Di Bartolomeo M, Borgonovo K, et al. Predictive role of BRAF mutations in patients with advanced colorectal cancer receiving cetuximab and panitumumab: a meta-analysis. Eur J Cancer 2015;51:587-94.

22. Rowland A, Dias MM, Wiese MD, Kichenadasse G, McKinnon RA, et al. Meta-analysis of BRAF mutation as a predictive biomarker of benefit from anti-EGFR monoclonal antibody therapy for RAS wild-type metastatic colorectal cancer. Br J Cancer 2015;112:1888-94.

23. Soeda H, Shimodaira H, Watanabe M, Suzuki T, Gamoh M, et al. Clinical usefulness of KRAS, BRAF, and PIK3CA mutations as predictive markers of cetuximab efficacy in irinotecan- and oxaliplatin-refractory Japanese patients with metastatic colorectal cancer. Int J Clin Oncol 2013;18:670-7.

24. Molinari F, Felicioni L, Buscarino M, De Dosso S, Buttitta F, et al. Increased detection sensitivity for KRAS mutations enhances the prediction of anti-EGFR monoclonal antibody resistance in metastatic colorectal cancer. Clin Cancer Res 2011;17:4901-14.

25. Saridaki Z, Tzardi M, Papadaki C, Sfakianaki M, Pega F, et al. Impact of KRAS, BRAF, PIK3CA mutations, PTEN, AREG, EREG expression and skin rash in $>/=2$ line cetuximab-based therapy of colorectal cancer patients. PLoS One 2011;6:e15980.

26. De Roock W, Claes B, Bernasconi D, De Schutter J, Biesmans B, et al. Effects of KRAS, BRAF, NRAS, and PIK3CA mutations on the efficacy of cetuximab plus chemotherapy in chemotherapy-refractory metastatic colorectal cancer: a retrospective consortium analysis. Lancet Oncol 2010;11:753-62.

27. Perrone F, Lampis A, Orsenigo M, Di Bartolomeo M, Gevorgyan A, et al. PI3KCA/PTEN deregulation contributes to impaired responses to cetuximab in metastatic colorectal cancer patients. Ann Oncol 2009;20:84-90.

28. Therkildsen C, Bergmann TK, Henrichsen-Schnack T, Ladelund S, Nilbert M. The predictive value of KRAS, NRAS, BRAF, PIK3CA and PTEN for anti-EGFR treatment in metastatic colorectal cancer: a systematic review and meta-analysis. Acta Oncol 2014;53:852-64.

29. Mohamed A, Twardy B, AbdAllah N, Akhras A, Ismail H, et al. Clinical impact of PI3K/BRAF mutations in RAS wild metastatic colorectal cancer: meta-analysis results. J Gastrointest Cancer 2018; doi: 10.1007/s12029-018-0062-y.

30. Ciardiello F, Kim N, Saeki T, Dono R, Persico MG, et al. Differential expression of epidermal growth factor-related proteins in human colorectal tumors. Proc Natl Acad Sci U S A 1991;88:7792-6.

31. Li XD, Miao SY, Wang GL, Yang L, Shu YQ, et al. Amphiregulin and epiregulin expression in colorectal carcinoma and the correlation with clinicopathological characteristics. Onkologie 2010;33:353-8.

32. Chayangsu C, Khunsri S, Sriuranpong V, Tanasanvimon S. The correlations between serum amphiregulin and other clinicopathological factors in colorectal cancer. J Gastrointest Oncol 2017;8:980-4.

33. Kuramochi H, Nakajima G, Kaneko Y, Nakamura A, Inoue Y, et al. Amphiregulin and epiregulin mRNA expression in primary colorectal cancer and corresponding liver metastases. BMC Cancer 2012;12:88.

34. Oliveras-Ferraros C, Cufi S, Queralt B, Vazquez-Martin A, Martin-Castillo B, et al. Cross-suppression of EGFR ligands amphiregulin and epiregulin and de-repression of FGFR3 signalling contribute to cetuximab resistance in wild-type KRAS tumour cells. Br J Cancer 2012;106:1406-14.

35. Johnson GR, Saeki T, Gordon AW, Shoyab M, Salomon DS, et al. Autocrine action of amphiregulin in a colon carcinoma cell line and immunocytochemical localization of amphiregulin in human colon. J Cell Biol 1992;118:741-51.

36. Khambata-Ford S, Garrett CR, Meropol NJ, Basik M, Harbison CT, et al. Expression of epiregulin and amphiregulin and K-ras mutation status predict disease control in metastatic colorectal cancer patients treated with cetuximab. J Clin Oncol 2007;25:3230-7. 
37. Jacobs B, De Roock W, Piessevaux H, Van Oirbeek R, Biesmans B, et al. Amphiregulin and epiregulin mRNA expression in primary tumors predicts outcome in metastatic colorectal cancer treated with cetuximab. J Clin Oncol 2009;27:5068-74.

38. Jing C, Jin YH, You Z, Qiong Q, Jun Z. Prognostic value of amphiregulin and epiregulin mRNA expression in metastatic colorectal cancer patients. Oncotarget 2016;7:55890-9.

39. Jonker DJ, Karapetis CS, Harbison C, O'Callaghan CJ, Tu D, et al. Epiregulin gene expression as a biomarker of benefit from cetuximab in the treatment of advanced colorectal cancer. Br J Cancer 2014;110:648-55.

40. Seligmann JF, Elliott F, Richman SD, Jacobs B, Hemmings G, et al. Combined epiregulin and amphiregulin expression levels as a predictive biomarker for panitumumab therapy benefit or lack of benefit in patients with RAS wild-type advanced colorectal cancer. JAMA Oncol 201610.1001/jamaoncol.2015.6065.

41. Venderbosch S, Nagtegaal ID, Maughan TS, Smith CG, Cheadle JP, et al. Mismatch repair status and BRAF mutation status in metastatic colorectal cancer patients: a pooled analysis of the CAIRO, CAIRO2, COIN, and FOCUS studies. Clin Cancer Res 2014;20:5322-30.

42. Roth AD, Tejpar S, Delorenzi M, Yan P, Fiocca R, et al. Prognostic role of KRAS and BRAF in stage II and III resected colon cancer: results of the translational study on the PETACC-3, EORTC 40993, SAKK 60-00 trial. J Clin Oncol 2010;28:466-74.

43. Popat S, Hubner R, Houlston RS. Systematic review of microsatellite instability and colorectal cancer prognosis. J Clin Oncol 2005;23:609-18.

44. Benatti P, Gafa R, Barana D, Marino M, Scarselli A, et al. Microsatellite instability and colorectal cancer prognosis. Clin Cancer Res 2005;11:8332-40.

45. Des Guetz G, Uzzan B, Nicolas P, Schischmanoff O, Perret GY, et al. Microsatellite instability does not predict the efficacy of chemotherapy in metastatic colorectal cancer. A systematic review and meta-analysis. Anticancer Res 2009;29:1615-20.

46. Topalian SL, Hodi FS, Brahmer JR, Gettinger SN, Smith DC, et al. Safety, activity, and immune correlates of anti-PD-1 antibody in cancer. N Engl J Med 2012;366:2443-54.

47. Brahmer JR, Drake CG, Wollner I, Powderly JD, Picus J, et al. Phase I study of single-agent anti-programmed death-1 (MDX-1106) in refractory solid tumors: safety, clinical activity, pharmacodynamics, and immunologic correlates. J Clin Oncol 2010;28:3167-75.

48. Le DT, Uram JN, Wang H, Bartlett BR, Kemberling H, et al. PD-1 blockade in tumors with mismatch-repair deficiency. N Engl J Med 2015;372:2509-20.

49. Overman MJ, Lonardi S, Wong KYM, Lenz HJ, Gelsomino F, et al. Durable clinical benefit with nivolumab plus ipilimumab in DNA mismatch repair-deficient/microsatellite instability-high metastatic colorectal cancer. J Clin Oncol 2018;36:773-9.

50. Rosenbaum MW, Bledsoe JR, Morales-Oyarvide V, Huynh TG, Mino-Kenudson M. PD-L1 expression in colorectal cancer is associated with microsatellite instability, BRAF mutation, medullary morphology and cytotoxic tumor-infiltrating lymphocytes. Mod Pathol 2016;29:1104-12.

51. Lee LH, Cavalcanti MS, Segal NH, Hechtman JF, Weiser MR, et al. Patterns and prognostic relevance of PD-1 and PD-L1 expression in colorectal carcinoma. Mod Pathol 2016;29:1433-42.

52. Gatalica Z, Snyder C, Maney T, Ghazalpour A, Holterman DA, et al. Programmed cell death 1 (PD-1) and its ligand (PD-L1) in common cancers and their correlation with molecular cancer type. Cancer Epidemiol Biomarkers Prev 2014;23:2965-70.

53. Droeser RA, Hirt C, Viehl CT, Frey DM, Nebiker C, et al. Clinical impact of programmed cell death ligand 1 expression in colorectal cancer. Eur J Cancer 2013;49:2233-42.

54. Wang HB, Yao H, Li CS, Liang LX, Zhang Y, et al. Rise of PD-L1 expression during metastasis of colorectal cancer: implications for immunotherapy. J Dig Dis 2017;18:574-81.

55. Lim YJ, Koh J, Kim S, Jeon SR, Chie EK, et al. Chemoradiation-induced alteration of programmed death-ligand 1 and CD8(+) tumorinfiltrating lymphocytes identified patients with poor prognosis in rectal cancer: a matched comparison analysis. Int J Radiat Oncol Biol Phys 2017;99:1216-24.

56. George TJ, Frampton GM, Sun J, Gowen K, Kennedy M, et al. Tumor mutational burden as a potential biomarker for PD1/PD-L1 therapy in colorectal cancer. J Clin Oncol 2016;34:abstr3587.

57. Ali SM, Gay LM, Elvin JA, Vergilio J, Ramkissoon SH, et al. MSI-high and MSI-stable colorectal carcinomas (CRC): a comprehensive genomic profiling (CGP) study. J Clin Oncol 2018;36:abstr3574.

58. Tran B, Kopetz S, Tie J, Gibbs P, Jiang ZQ, et al. Impact of BRAF mutation and microsatellite instability on the pattern of metastatic spread and prognosis in metastatic colorectal cancer. Cancer 2011;117:4623-32.

59. Tie J, Gibbs P, Lipton L, Christie M, Jorissen RN, et al. Optimizing targeted therapeutic development: analysis of a colorectal cancer patient population with the BRAF(V600E) mutation. Int J Cancer 2011;128:2075-84.

60. Davies H, Bignell GR, Cox C, Stephens P, Edkins S, et al. Mutations of the BRAF gene in human cancer. Nature 2002;417:949-54.

61. Clancy C, Burke JP, Kalady MF, Coffey JC. BRAF mutation is associated with distinct clinicopathological characteristics in colorectal cancer: a systematic review and meta-analysis. Colorectal Dis 2013;15:e711-8.

62. Chapman PB, Hauschild A, Robert C, Haanen JB, Ascierto P, et al. Improved survival with vemurafenib in melanoma with BRAF V600E mutation. N Engl J Med 2011;364:2507-16.

63. Hauschild A, Grob JJ, Demidov LV, Jouary T, Gutzmer R, et al. Dabrafenib in BRAF-mutated metastatic melanoma: a multicentre, openlabel, phase 3 randomised controlled trial. Lancet 2012;380:358-65.

64. Kopetz S, Desai J, Chan E, Hecht JR, O'Dwyer PJ, et al. Phase II pilot study of vemurafenib in patients with metastatic BRAF-mutated colorectal cancer. J Clin Oncol 2015;33:4032-8.

65. Hyman DM, Puzanov I, Subbiah V, Faris JE, Chau I, et al. Vemurafenib in multiple nonmelanoma cancers with BRAF V600 mutations. N Engl J Med 2015;373:726-36.

66. Prahallad A, Sun C, Huang S, Di Nicolantonio F, Salazar R, et al. Unresponsiveness of colon cancer to BRAF(V600E) inhibition through feedback activation of EGFR. Nature 2012;483:100-3.

67. Kopetz C, McDonough SL, Morris VK, Lenz HJ, Magliocco AM, et al. Randomized trial of irinotecan and cetuximab with or without vemurafenib in BRAF-mutant metastatic colorectal cancer (SWOG 1406). J Clin Oncol 2017;35;abstr520. 
68. van Geel R, Tabernero J, Elez E, Bendell JC, Spreafico A, et al. A phase Ib dose-escalation study of Encorafenib and Cetuximab with or without alpelisib in metastatic BRAF-mutant colorectal cancer. Cancer Discov 2017;7:610-9.

69. Atreya CE, Van Cutsem E, Bendell JC, Andre T, Schellens JHM, et al. Updated efficacy of the MEK inhibitor trametinib (T), BRAF inhibitor dabrafenib (D), and anti-EGFR antibody panitumumab (P) in patients (pts) with BRAF V600E mutated (BRAFm) metastatic colorectal cancer (mCRC). J Clin Oncol 2015;33:abstr103.

70. Richman SD, Southward K, Chambers P, Cross D, Barrett J, et al. HER2 overexpression and amplification as a potential therapeutic target in colorectal cancer: analysis of 3256 patients enrolled in the QUASAR, FOCUS and PICCOLO colorectal cancer trials. J Pathol 2016;238:562-70.

71. Lee WS, Park YH, Lee JN, Baek JH, Lee TH, et al. Comparison of HER2 expression between primary colorectal cancer and their corresponding metastases. Cancer Med 2014;3:674-80.

72. Takegawa N, Yonesaka K, Sakai K, Ueda H, Watanabe S, et al. HER2 genomic amplification in circulating tumor DNA from patients with cetuximab-resistant colorectal cancer. Oncotarget 2016;7:3453-60.

73. Valtorta E, Martino C, Sartore-Bianchi A, Penaullt-Llorca F, Viale G, et al. Assessment of a HER2 scoring system for colorectal cancer: results from a validation study. Mod Pathol 2015;28:1481-91.

74. Hurwitz H, Raghav KPS, Burris HA, Kurzrock R, Sweeney C, et al. Pertuzumab + trastuzumab for HER2-amplified/overexpressed metastatic colorectal cancer (mCRC): Interim data from MyPathway. J Clin Oncol 2017;35:abstr676.

75. Pietrantonio F, Di Nicolantonio F, Schrock AB, Lee J, Tejpar S, et al. ALK, ROS1, and NTRK rearrangements in metastatic colorectal cancer. J Natl Cancer Inst 2017;109.

76. Drilon A, Siena S, Ou SI, Patel M, Ahn MJ, et al. Safety and antitumor activity of the multitargeted Pan-TRK, ROS1, and ALK inhibitor entrectinib: combined results from two phase i trials (ALKA-372-001 and STARTRK-1). Cancer Discov 2017;7:400-9.

77. Amatu A, Somaschini A, Cerea G, Bosotti R, Valtorta E, et al. Novel CAD-ALK gene rearrangement is drugable by entrectinib in colorectal cancer. Br J Cancer 2015;113:1730-4.

78. Sartore-Bianchi A, Ardini E, Bosotti R, Amatu A, Valtorta E, et al. Sensitivity to entrectinib associated with a novel LMNA-NTRK1 gene fusion in metastatic colorectal cancer. J Natl Cancer Inst 2016;108.

79. Brannon AR, Vakiani E, Sylvester BE, Scott SN, McDermott G, et al. Comparative sequencing analysis reveals high genomic concordance between matched primary and metastatic colorectal cancer lesions. Genome Biol 2014;15:454.

80. Vakiani E, Janakiraman M, Shen R, Sinha R, Zeng Z, et al. Comparative genomic analysis of primary versus metastatic colorectal carcinomas. J Clin Oncol 2012;30:2956-62.

81. Bertucci F, Finetti P, Guille A, Adelaide J, Garnier S, et al. Comparative genomic analysis of primary tumors and metastases in breast cancer. Oncotarget 2016;7:27208-19.

82. Haraldsdottir S, Roth R, Pearlman R, Hampel H, Arnold CA, et al. Mismatch repair deficiency concordance between primary colorectal cancer and corresponding metastasis. Fam Cancer 2016;15:253-60.

83. Jung J, Kang Y, Lee YJ, Kim E, Ahn B, et al. Comparison of the mismatch repair system between primary and metastatic colorectal cancers using immunohistochemistry. J Pathol Transl Med 2017;51:129-36.

84. Agoston EI, Baranyai Z, Dede K, Bodoky G, Kulka J, et al. Occurrence, intratumoral heterogeneity, prognostic and predictive potential of microsatellite instability following surgical resection of primary colorectal carcinomas and corresponding liver metastases. Orv Hetil 2015;156:1460-71.

85. Spindler KL, Pallisgaard N, Andersen RF, Jakobsen A. Changes in mutational status during third-line treatment for metastatic colorectal cancer--results of consecutive measurement of cell free DNA, KRAS and BRAF in the plasma. Int J Cancer 2014;135:2215-22.

86. Thierry AR, Mouliere F, El Messaoudi S, Mollevi C, Lopez-Crapez E, et al. Clinical validation of the detection of KRAS and BRAF mutations from circulating tumor DNA. Nat Med 2014;20:430-5.

87. Bachet JB, Bouche O, Taieb J, Dubreuil O, Garcia ML, et al. RAS mutation analysis in circulating tumor DNA from patients with metastatic colorectal cancer: the AGEO RASANC prospective multicenter study. Ann Oncol 2018;29:1211-9.

88. Tie J, Kinde I, Wang Y, Wong HL, Roebert J, et al. Circulating tumor DNA as an early marker of therapeutic response in patients with metastatic colorectal cancer. Ann Oncol 2015;26:1715-22.

89. Garlan F, Laurent-Puig P, Sefrioui D, Siauve N, Didelot A, et al. Early evaluation of circulating tumor dna as marker of therapeutic efficacy in metastatic colorectal cancer patients (PLACOL study). Clin Cancer Res 2017;23:5416-25.

90. Diaz LA, Jr., Williams RT, Wu J, Kinde I, Hecht JR, et al. The molecular evolution of acquired resistance to targeted EGFR blockade in colorectal cancers. Nature 2012;486:537-40.

91. Guinney J, Dienstmann R, Wang X, de Reynies A, Schlicker A, et al. The consensus molecular subtypes of colorectal cancer. Nat Med 2015;21:1350-6. 